\title{
Conhecimentos sobre o serviço de atendimento móvel de urgência (SAMU) da população de três municípios com realidades distintas
}

\author{
Knowledge about the emergency medical services (EMS) of the population \\ of three cities with different realities \\ Conocimiento acerca de lo servicio móvil de urgencia (SAMU) de la población de \\ municipios con diferentes realidades
}

\author{
Marina Coimbra da $\mathbf{C R U Z}^{1}$ \\ Renan Paes de CAMARGO ${ }^{1}$ \\ Augusto Séttemo FERREIRA \\ Mateus Tomáz BORGES ${ }^{1}$ \\ Marlene Cabral Coimbra da $\mathbf{C R U Z}^{2}$ \\ Luciana Estevam SIMONATO²

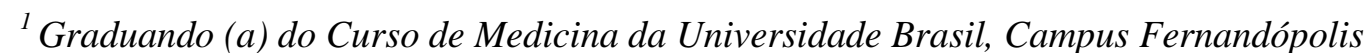 \\ 15600-000 Fernandópolis-SP, Brasil \\ ${ }^{2}$ Docente dos Cursos de Medicina e Odontologia da Universidade Brasil, Campus Fernandópolis \\ 15600-000 Fernandópolis-SP, Brasil
}

\begin{abstract}
Resumo
Introdução: O atendimento pré-hospitalar móvel é prestado pelo Serviço de Atendimento Móvel às Urgências, o qual funciona 24 horas por dia e apresenta três objetivos básicos: atender agravos agudos; regular o sistema de vagas de urgência e emergência em hospitais e educar em urgência e emergência. O contato é feito gratuitamente pelo número 192. Os problemas presentes no serviço estão relacionados principalmente, à demanda inadequada, pois a população é quem julga subjetivamente o que considera urgente. Diante deste fato, torna-se relevante avaliar o conhecimento da população em geral sobre o serviço. Objetivo: Avaliar saberes de população acerca do Serviço de Atendimento Móvel de Urgência. Material e Método: Estudo transversal, realizado através da aplicação de questionário a populares de três municípios. Resultados: Foram entrevistados 257 populares, sendo que a maioria apresentou conhecimento sobre a presença ou não do serviço em seu município; 44\% sabiam o telefone, com o contato 193 assinalado em 14,4\% dos casos; $13 \%$ dos moradores de Estrela D’Oeste afirmaram já terem acionado o Serviço de Atendimento Móvel às Urgências, contra 46,7\% em Fernandópolis; $84 \%$ tinham ciência de seu caráter emergencial; 70,4\% tiveram acertos sobre emergências psiquiátricas; $42 \%$ acreditam que o aparelho serve como meio de transporte; $62 \%$ sabiam das informações a serem fornecidas e quase a totalidade compreende que seu financiamento é público. Conclusão: O conhecimento da população foi razoável, no entanto, tal desempenho não deve ser tido como suficiente, uma vez que qualquer erro ou delonga na solicitação do serviço pode trazer sequelas ou mortes.

Descritores: Criança Hospitalizada; Adolescente Hospitalizado; Saúde Bucal; Odontologia Preventiva; Assistência Odontológica.
\end{abstract}

\begin{abstract}
Introduction: Prehospital mobile care is provided by the Emergency Medical Services, which operates 24 hours a day and has three basic objectives: to treat acute conditions; regulate the system of emergency vacancies in hospitals and educate about emergency situations. The contact is made freely through the number 192. The problems present in the service are mainly related to the inadequate demand, because the population is the one who judges subjectively what it considers urgent. Given this fact, it is relevant to evaluate the knowledge of the general population about the service. Objective: Evaluate people's knowledge about the Emergency Medical Services. Material and Method: Cross-sectional study, performed through the application of questionnaire to popular of three cities. Results: A total of 257 people were interviewed, most of whom presented knowledge about the presence or not of this service in the city; $44 \%$ knew the contact of the service, with 193 contact indicated in $14.4 \%$ of cases; $13 \%$ of the residents of Estrela D'Oeste stated they had already contacted the Emergency Medical Service, against $46.7 \%$ in Fernandópolis; $84 \%$ were aware of the emergency feature; $70.4 \%$ knew the service is responsible of psychiatric emergencies; $42 \%$ believe that service operate as a way of transportation; $62 \%$ knew the information to be provided and almost all of the participants are aware of its public funding. Conclusion: Popular knowledge was reasonable; however, such performance is not enough, since error or delay in requesting the service can culminate in sequelae or death.

Descriptors: Emergency Medical Services; First Aid; Health Services.
\end{abstract}

\section{Resumen}

Introducción: La atención prehospitalaria móvil es proporcionada por el Servicio Móvil de Urgencia, que opera 24 horas al día y tiene tres objetivos básicos: ayudar em las enfermedades agudas; regular el sistema de vacantes de emergencia en los hospitales y la educación en las situaciones de emergencia. El contacto se hace gratis por el número 192. Su problemas actuales están relacionados principalmente a la demanda inadecuada debido a que la población que subjetivamente jueces que considera urgente. Teniendo en cuenta este hecho, es relevante para evaluar el conocimiento de la población en general sobre el servicio. Objetivos: Evaluar el conocimiento de la población sobre el Servicio Móvil de Urgencia. Material y Métodos: Estudio transversal a través de cuestionario aplicado a populares de tres municipios. Resultados: Se entrevistó 257 populares; la mayoría tenía conocimiento de la presencia o no del servicio en la ciudad; 44\% sabían el contacto del servicio; con el número 193 marcado 14,4\% del tiempo; 13\% de los residentes de Estrela D'Oeste, dijo que ya han contactado el Servicio Móvil de Urgencia, frente al 46,7\% en Fernandópolis; $84 \%$ eran conscientes de su función de emergencia; 70,4\% conocían que el servicio es responsable por las emergencias psiquiátricas; $42 \%$ cree que el servicio sirve como transporte; $62 \%$ conocían la información que debe proporcionarse y comprende que la financiación es pública. Conclusión: El conocimiento era razonable, sin embargo, este resultado no debe tomarse como suficiente, ya que cualquier error o demora en la solicitud del servicio puede traer consecuencias o muerte.

Descriptores: Servicios Médicos de Urgencia; Primeros Auxilios; Servicios de Salud.

\section{INTRODUÇÃO}

Nos últimos anos, o Brasil vem sofrendo uma série de mudanças socioeconômicas e demográficas que acabam por interferir no processo saúde-doença. Estas alterações estão fazendo com que ocorra um maior número de eventos urgentes, exigindo grande eficiência dos serviços que lidam com tais situações. Diante disso, aumentou-se a pressão sobre os serviços de emergência médica e gerou-se uma necessidade de atendimento imediato no local de ocorrência 
e posterior transporte para um serviço de atendimento definitivo. Assim, surgiram os Serviços de Atendimento PréHospitalar (SvAPH), que atualmente recebem a denominação de Atendimento Pré-Hospitalar (APH), cujo objetivo é uma intervenção precoce, capaz de reduzir índices de mortalidade e possíveis sequelas ${ }^{1,2}$.

Atualmente, o atendimento pré-hospitalar móvel no Brasil é prestado principalmente pelo Serviço de Atendimento Móvel de Urgência (SAMU), implementado em 2003, após um acordo bilateral com a França, e oficializado em 2004 pelo Decreto $\mathrm{n}^{\circ} 5.055^{3}$. Para tanto, foi formulada, em 2003, a Política Nacional de Atenção às Urgências ${ }^{4}$, norteada pelos princípios do Sistema Único de Saúde (SUS) e baseada em cinco eixos: "promoção da qualidade de vida, organização em rede, operação de centrais de regulação, capacitação e educação continuada e humanização da atenção, ".

Com tais ações, o serviço almeja diminuir o número de mortes, reduzir o tempo de internação e evitar as possíveis sequelas decorrentes da demora na realização do atendimento pré-hospitalar ${ }^{6}$. Segundo a Sala de Apoio à Gestão Estratégica (SAGE) ${ }^{7}$ do Ministério da Saúde (MS), em 2015 foram investidos 1 bilhão de reais no custeio do SAMU no país. Esse investimento no serviço de urgência é uma mudança na tradição dos governos, que costumavam dar mais prioridade às políticas de saúde voltadas às crianças e às mães ${ }^{8}$.

O SAMU brasileiro baseia-se no modelo francês de urgências denominado de Service d'Aide Médicale d'Urgence, também através da sigla SAMU, que é considerado o melhor do mundo por diversos especialistas, o qual preconiza o atendimento na cena do acontecimento ${ }^{9,10}$. Difere, no entanto, em situações de menor complexidade, por utilizar enfermeiros e técnicos de enfermagem sem a presença de um médico ${ }^{9}$. É administrado pela Prefeitura, por meio da Secretaria Municipal de Saúde, em parceria com o $\mathrm{MS}^{10}$. Seu funcionamento é 24 horas por dia e abrange uma área geográfica específica. Apresenta três objetivos básicos: atender agravos agudos de natureza clínica, psiquiátrica, cirúrgica, traumática, obstétrica e ginecológica; regular o sistema de vagas de urgência e emergência em hospitais secundários e terciários por uma central 24 horas e educar em urgência e emergência ${ }^{6,10}$. O contato é feito por meio da ligação gratuita para o número 192, em que, em um primeiro momento, um atendente identifica a solicitação e, na sequência, passa o caso para o médico regulador, que analisará qual o grau de urgência e tomará a decisão mais plausível para o caso, podendo ser desde uma simples conversa de aconselhamento médico até o envio de uma Unidade de Suporte Avançado de Vida ${ }^{6}$. Em 2016, abarcava cerca de 157 milhões de pessoas, o correspondente a 77,5\% da população brasileira. Contando com um total de 3.354 veículos, sendo 2.525 ambulâncias básicas, 583 ambulâncias de suporte avançado, 226 motolâncias, 13 embarcações e 7 aeromédicos ${ }^{7}$.

Os problemas presentes no serviço estão relacionados à demora no transporte aos hospitais, onde acontecerão os atendimentos finais e, principalmente, à busca inadequada do SAMU pela população, visto que as próprias pessoas acabam julgando o que é urgente ou não, além de que muitos querem ser atendidos visando a uma resolução mais rápida de suas queixas, gerando uma sobrecarga do sistema ${ }^{5,9,11}$

No município de Fernandópolis, interior de São Paulo, formado por 67.836 habitantes, o SAMU apresenta ação regional, através de uma Central de Regulação de Urgência, contando com três ambulâncias básicas e uma avançada para o atendimento de uma região constituída por 13 municípios (Fernandópolis, São João das Duas Pontes, Estrela D’Oeste, Pedranópolis, Mira Estrela, Macedônia, Turmalina, Guarani D’Oeste, Populina, Ouroeste, Indiaporã e Meridiano) ${ }^{7}$

Estrela D'Oeste, também no interior de São Paulo, apresenta 8.460 habitantes, e não possui uma unidade do SAMU, no entanto recebe seus serviços através da Central de Regulação de Urgência do SAMU Fernandópolis ${ }^{7}$.

Por outro lado Pirajuba, no interior de Minas Gerais, apresenta uma população de 5.396 habitantes, não possui nem recebe os serviços do SAMU de nenhuma Central de Regulação de Urgência de sua região ${ }^{7}$.

Diante da relevância do assunto, o presente estudo objetivou avaliar o conhecimento da população em geral acerca do SAMU-192, sua ação, abrangência e acionamento, bem como uso prévio do serviço.

\section{MATERIAL E MÉTODO}

Trata-se de um estudo descritivo, do tipo transversal, transcorrido em 3 municípios distintos: Fernandópolis/SP, que apresenta uma central do SAMU; Estrela D'Oeste/SP, que recebe o atendimento do SAMU pela central regional de Fernandópolis; e Pirajuba/MG, a qual não é abrangida pelos serviços do SAMU. Foi submetido à avaliação pelo Comitê de Ética em Pesquisa da Universidade Brasil, sob o CAAE 52671215.4.0000.5494.

O estudo efetuou-se mediante a aplicação de um questionário estruturado com 13 questões de múltipla escolha, incluindo dados de identificação educacional, de gênero e de idade, para levantamento sobre o conhecimento acerca do SAMU e de seu funcionamento.

A escolha dos participantes deu-se de forma aleatória, porém se respeitaram os critérios de inclusão: ter idade igual ou superior a 18 anos e ser residente de Fernandópolis, Estrela D'Oeste ou Pirajuba. As entrevistas foram realizadas de forma aleatória com populares dos 3 municípios abrangidos, em locais de grande circulação de pessoas, em que estes eram abordados, explicitados sobre a pesquisa e convidados a participar da mesma. Os casos afirmativos assinavam um Termo De Consentimento Livre Esclarecido e respondiam o questionário de forma sigilosa.

A amostra foi composta por 257 pessoas, sendo 84 de Pirajuba/MG, 84 de Estrela D’Oeste/SP e 89 de Fernandópolis/SP

Os dados coletados foram agrupados de acordo com o gênero, a idade, o grau de escolaridade, cidade em que reside e uso prévio do serviço, analisados e os resultados foram apresentados de forma absoluta e relativa, em tabelas e gráficos.

\section{RESULTADOS}

Dos 257 populares que responderam o questionário, 158 eram mulheres $(61,5 \%)$ e 98 homens $(38,1 \%)$, sendo que um dos entrevistados não identificou seu sexo. Observou-se predomínio na faixa etária dos 20 aos 29 anos $(28,8 \%)$ e do nível escolar médio $(46,3 \%)$, sendo que um popular não relatou seu grau de escolaridade. Os dados das variáveis demográficas estão expostos na Tabela 1.

Em relação ao número de contato do SAMU-192, conforme dados da Tabela 2, de uma maneira geral, $44 \%$ dos entrevistados detinham um conhecimento errôneo ou não souberam informar o telefone do serviço. Os moradores de Fernandópolis, que apresenta uma unidade do SAMU, destacaram-se, pois $72 \%$ de seus entrevistados sabiam o 
contato 192. Embora Estrela D'Oeste receba atendimento do SAMU e Pirajuba não, os dados mostraram uma inversão no previsto, uma vez que os pirajubenses participantes detinham mais conhecimento sobre o contato do SAMU $(51,2 \%)$ do que os entrevistados munícipes de Estrela D'Oeste $(44,1 \%)$.

Tabela 1. Descrição da população abordada quanto ao grau de escolaridade, sexo e idade

\begin{tabular}{|c|c|c|c|c|}
\hline Variável & $\begin{array}{l}\text { Estrela } \\
\text { D'oeste }\end{array}$ & Fernandópolis & Pirajuba & Total \\
\hline \multicolumn{5}{|l|}{ Escolaridade: } \\
\hline Fundamental & $29(34,5 \%)$ & $16(18,0 \%)$ & $17(20,2 \%)$ & $62(24,1 \%)$ \\
\hline Médio & $32(38,1 \%)$ & $44(49,4 \%)$ & $43(51,2 \%)$ & $119(46,3 \%)$ \\
\hline Superior & $22(26,2 \%)$ & $29(32,6 \%)$ & $24(28,6 \%)$ & $75(29,2 \%)$ \\
\hline Não Respondeu & $1(1,2 \%)$ & & & $1(0,4 \%)$ \\
\hline \multicolumn{5}{|l|}{ Sexo: } \\
\hline Feminino & $58(69,0 \%)$ & $61(68,5 \%)$ & $39(46,4 \%)$ & $158(61,5 \%)$ \\
\hline Masculino & $26(31,0 \%)$ & $28(31,5 \%)$ & $44(52,4 \%)$ & $98(38,1 \%)$ \\
\hline Não Respondeu & & & $1(1,2 \%)$ & $1(0,4 \%)$ \\
\hline \multicolumn{5}{|l|}{ Idade: } \\
\hline $18-19$ Anos & $6(7,1 \%)$ & $5(5,6 \%)$ & $6(7,1 \%)$ & $17(6,6 \%)$ \\
\hline $20-29$ Anos & $19(22,6 \%)$ & $29(32,6 \%)$ & $26(31,0 \%)$ & $74(28,8 \%)$ \\
\hline $30-39$ Anos & $7(8,3 \%)$ & $22(24,7 \%)$ & $9(10,7 \%)$ & $38(14,8 \%)$ \\
\hline $40-49$ Anos & $25(30,0 \%)$ & $16(18,0 \%)$ & $23(27,4 \%)$ & $64(25,0 \%)$ \\
\hline $50-59$ Anos & $9(10,7 \%)$ & $12(13,5 \%)$ & $12(14,3 \%)$ & $33(12,8 \%)$ \\
\hline 60 anos ou mais & $18(21,4 \%)$ & $5(5,6 \%)$ & $8(9,5 \%)$ & $31(12,0 \%)$ \\
\hline
\end{tabular}

Tabela 2. Distribuição dos acertos quanto ao telefone para contanto do SAMU-192

\begin{tabular}{c|c|c|c|c|c|c}
\hline \multirow{2}{*}{ CIDADE } & \multicolumn{6}{|c}{ CONTATO DO SAMU } \\
\cline { 2 - 7 } & \multicolumn{2}{|c|}{192} & \multicolumn{2}{c}{ OUTRO } & \multicolumn{2}{c}{ NÃO SEI } \\
\cline { 2 - 7 } & $\mathbf{n}$ & $\%$ & $\mathbf{n}$ & $\%$ & $\mathbf{n}$ & $\%$ \\
\hline ESTRELA D'OESTE & 37 & 44,1 & 18 & 21,4 & 29 & 34,5 \\
FERNANDÓPOLIS & 64 & 72,0 & 20 & 22,4 & 5 & 5,6 \\
PIRAJUBA & 43 & 51,2 & 9 & 10,7 & 32 & 38,1 \\
TOTAL & 144 & 56,0 & 47 & 18,3 & 66 & 25,7 \\
\hline
\end{tabular}

A análise da porcentagem de acertos obtidos no quesito "contato do SAMU-192" relativamente ao sexo mostrou que as mulheres abordadas apresentavam maior conhecimento que os homens, $65 \%$ contra $57 \%$, respectivamente. Não houve relação entre porcentagem de acerto e maior nível de escolaridade, uma vez que os entrevistados com ensino médio foram os que obtiveram maior porcentagem de acerto (77\%), embora o grau fundamental tenha revelado menor conhecimento, com apenas $37,7 \%$ de acertos. Com relação à idade, a terceira década de vida foi a maior detentora do contato dentre os participantes, com $68 \%$ assinalando o contato 192; enquanto que os entrevistados com 60 anos ou mais foram os que menos distinguiram corretamente o telefone do SAMU, expondo $58 \%$ de erro.

Dentre outros telefones assinalados como sendo do SAMU, destaca-se o contato 193, com 14,4\% de frequência.

Avaliou-se o conhecimento da existência ou não de uma unidade do SAMU em cada município e, considerandose que Fernandópolis apresenta uma Central Regional enquanto que Estrela D'Oeste e Pirajuba não. Verificou-se que, de maneira geral, a população é consciente da presença ou não do SAMU em sua cidade, sendo as porcentagens de acertos em Estrela D'Oeste, Fernandópolis e Pirajuba de $90,5 \%, 98,9 \%$ e $89,2 \%$, respectivamente.

Quanto ao recebimento do SAMU através de uma Central Reguladora Regional (Figura 1) houve diferença significante entre Estrela D'Oeste e Pirajuba. A grande maioria $(90,4 \%)$ dos moradores de Estrela D'Oeste acertaram que a cidade recebe o serviço da Central Regional de Fernandópolis, contra apenas 53,5\% de acerto em Pirajuba, que não é coberta por este serviço médico móvel.

No que se refere ao uso prévio do serviço, como visto na Figura 2, apenas 11 residentes de Estrela D'Oeste (13\%) afirmaram já terem acionado o SAMU-192. No entanto, $37 \%$ destes responderam erroneamente o telefone para contato do aparelho. Já no município de Fernandópolis, $46,7 \%$ dos entrevistados disseram ter acionado o serviço ao menos uma vez e, destes, $84,2 \%$ sabiam o número de contato do mesmo. Desconsiderou-se esta questão no município de Pirajuba, visto que sua região não é abrangida pelo SAMU.

Quanto as situações pertinentes ao acionamento do SAMU, a maioria (84\%) dos participantes soube que o serviço atua em qualquer situação de doença ou acidente de caráter emergencial ou de urgência, sendo que os acertos foram maiores em Fernandópolis $(87,6 \%)$, seguido de Estrela D'Oeste $(84,5 \%)$ e Pirajuba $(79,7 \%)$.

$\mathrm{Na}$ Figura 3 podem ser observados os resultados referentes ao que a população acredita ser função do SAMU Emergências psiquiátricas foram listadas como encargo do SAMU pela maior parte dos residentes dos 3 municípios abrangidos, com uma porcentagem de acertos geral de $70,4 \%$. No entanto, $42 \%$ de todos os entrevistados demonstraram pensar que o SAMU funciona também como meio de transporte até centros de saúde, principalmente em Pirajuba, em que mais da metade dos participantes $(61,9 \%)$ tinham tal fato como verdadeiro.

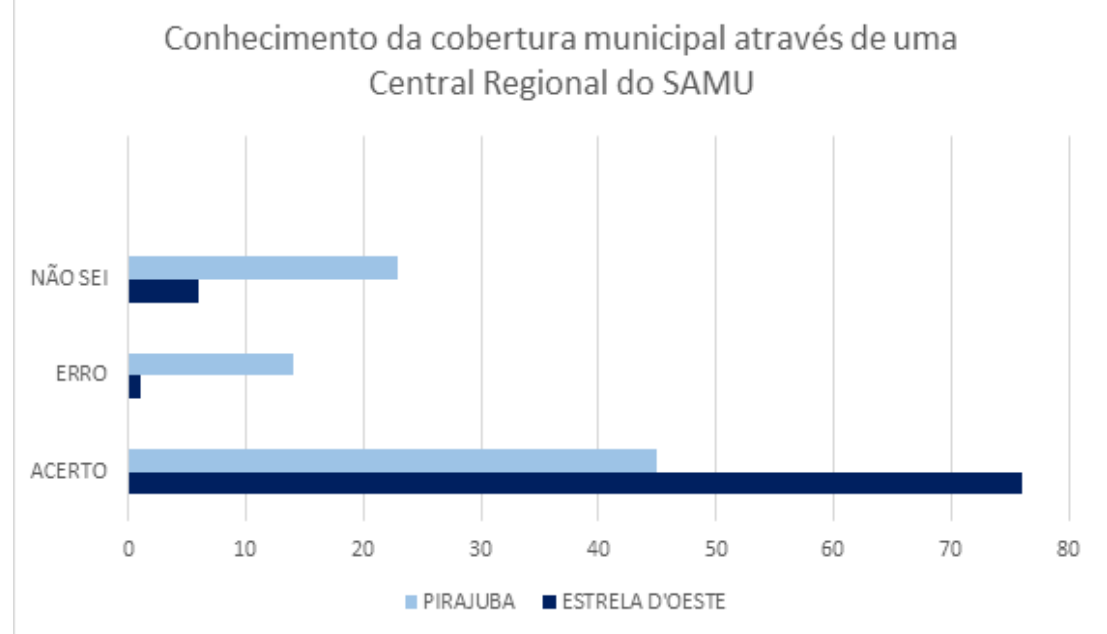

Figura 1: Conhecimento da cobertura municipal através de uma Central Regional do SAMU.

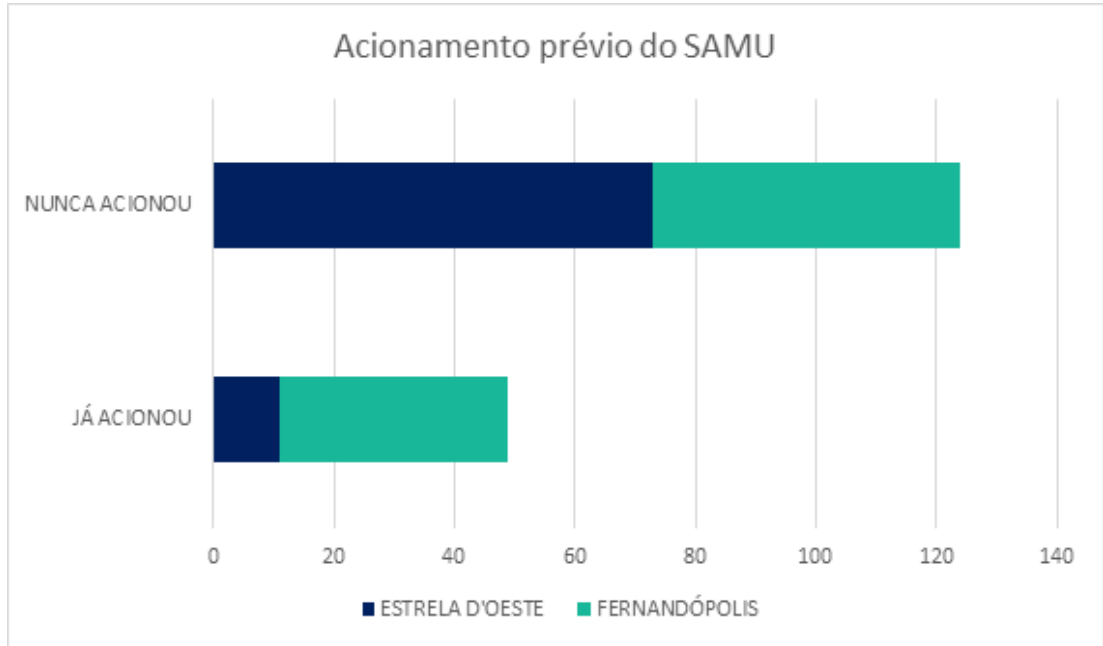

Figura 2: Acionamento prévio do SAMU.

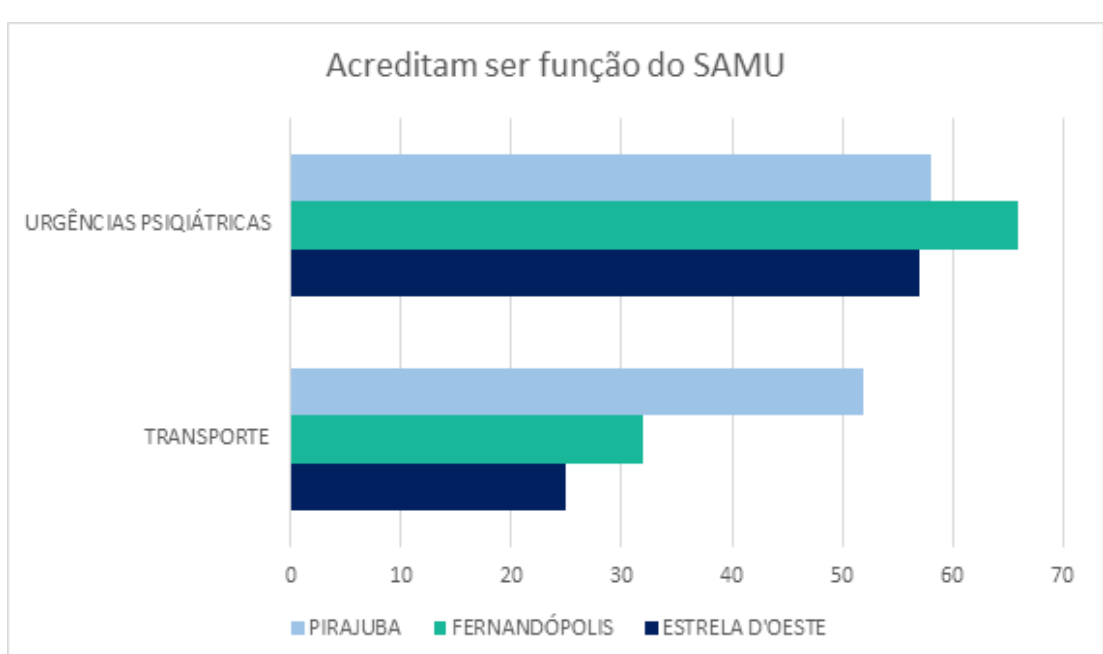

Figura 3: Ações que a população julga ser função do SAMU. 
Ao se acionar o SAMU, o regulador necessita de informações, a serem fornecidas pelo solicitante, sobre o ocorrido a fim de se avaliar a gravidade da situação para planejamento da atitude mais adequada ao caso. Ao se questionar quais são estas informações relevantes a serem relatadas, de maneira geral, 161 dos avaliados (62\%) souberam listar de forma completa tais dados. Pirajuba apresentou a maior porcentagem de acertos (68\%), seguida de Estrela D'Oeste (62\%) e Fernandópolis (58\%).

Quanto ao custeio do SAMU, a maioria dos entrevistados dos 3 municípios apresentou conhecimento de que seu financiamento é público: $98,8 \%$ dos residentes de Estrela D’Oeste; 98,9\% de Fernandópolis e 92,8\% de Pirajuba.

\section{DISCUSSÃO}

O conhecimento sobre o SAMU é de suma importância para que a população possa agir correta e rapidamente ao se deparar com uma situação de urgência e emergência e, consequentemente, usufruir de forma eficaz e benéfica do serviço ${ }^{6}$.

Segundo as diretrizes para emergências cardiovasculares da American Heart Association $(2010)^{12}$, a cadeia de sobrevida inicia-se pelo acionamento do serviço móvel de urgência, em especial quando houver um único socorrista. Tempo é vida, portanto é de extrema importância que a população como um todo saiba o telefone de contato do SAMU-192 para seu rápido acionamento, em especial nas situações de nervosismo que podem naturalmente atrapalhar a tomada de atitude e a evocação do contato quando este não estiver perfeitamente decorado. A delonga ou mesmo o não acionamento do serviço, pode resultar em sequelas ou diminuição da sobrevida das vítimas ${ }^{6}$. Estudos de Horta et al. $(2011)^{13}$ e Fernandes et al. $(2014)^{6}$ mostraram que nem mesmo graduandos do curso de medicina apresentavam conhecimento pleno sobre o número de acionamento do SAMU, em que $18,3 \%$ e $16,39 \%$, respectivamente, erraram o telefone 192. Pontes-Neto et al. $(2008)^{14}$ entrevistou aleatoriamente mais de 800 populares nas cidades de Fortaleza, Salvador, São Paulo e Ribeirão Preto e observou que apenas 34,6\% dos participantes reconheciam 192 e 193 como contatos para emergências médicas. O contato 193 , segundo mais listado no presente estudo $(14,4 \%)$, relacionase ao Corpo de Bombeiros da Polícia Militar, cuja função, neste caso, é "realizar busca, resgate e salvamento, nos casos de desastres, calamidades e outras situações de emergência"15. Logo, o contato 193 deve ser acionado em situações de risco em que haja a necessidade de resgate e salvamento; enquanto que o SAMU-192 deve ser solicitado em situações emergenciais de caráter clínico, psiquiátrico, cirúrgico, traumático, obstétrico e ginecológico ${ }^{6,10,15}$.

No presente trabalho, os resultados obtidos mostram que os entrevistados com 60 anos ou mais foram os que menos distinguiram corretamente o telefone do SAMU, expondo $58 \%$ de erro.

A distinção feita pelos usuários sobre o que é ou não urgente é subjetiva9. Dessa forma, a decisão sobre o acionamento do serviço fundamenta-se em experiências pessoais e em vivências de outros usuários. Tal fato, somado a angústia e a sensação de impotência frente ao sofrimento alheio, por vezes, resulta em demandas não pertinentes ${ }^{9,16}$. O foco de assistência do SAMU é voltado a situações urgentes de doença e agravos a saúde e quadros agudizados, não abrangendo acompanhamento de doenças crônicas ou casos de menor gravidade ${ }^{9,10}$. A falta de conhecimento sobre o funcionamento e encargos do SAMU, aliado a situações de vulnerabilidade social e emocional diante de casos de dor, podem resultar em aumento no número de solicitações não oportunas ao serviço, sobrecarregando financeiramente o sistema e podendo atrasar o atendimento a cenas cujo socorro é realmente necessário.

Cabe ao SAMU amparar urgências precocemente e no local do acontecimento, somente se incumbindo do transporte até um estabelecimento de saúde ou interhospitalar em casos necessários, de maior gravidade ou que apresentem risco de morte ${ }^{9,10,11}$. No entanto, estudos apontam, semelhantemente ao presente trabalho, que muitos usuários acreditam que o transporte à serviços de saúde seja competência do SAMU, em especial aqueles que estão em condição de vulnerabilidade social ${ }^{3,17}$. Cochlar $(2007)^{18}$ evidenciou que, em Canoas, $45,7 \%$ dos entrevistados acionariam o serviço para transportar pessoas com dificuldade para caminhar.

Outro ponto avaliado foi referente a atuação do SAMU frente a urgências psiquiátricas, em que a maioria dos entrevistados apresentou conhecimento satisfatório quanto a este tipo de abordagem do serviço. Em contrapartida, Cochlar $(2007)^{18}$ encontrou que somente 46,2\% dos usuários, em um município do Rio Grande do Sul, acionariam o SAMU em tentativas de suicídio. Horta et al. $(2011)^{13}$ observou que pouco mais da metade dos alunos de medicina entrevistados ligariam para o SAMU diante de um episódio de tentativa de suicídio. Um estudo que avaliou a pertinência das solicitações do serviço do SAMU em Porto Alegre demonstrou a subjetividade envolvendo a percepção de urgência, uma vez que tentativa de suicídio foi considerada ora pertinente, ora não pertinente à atuação do serviço ${ }^{9}$. Jardim $(2008)^{19}$ verificou que o SAMU representa possibilidades ao atendimento psiquiátrico em momentos de crise, visto que se desloca ao local da vítima, garantindo rapidez no atendimento, o que pode evitar internações, além de viabilizar a inclusão familiar no tratamento e encaminhamentos mais conexos. No entanto, observou também que o serviço apresenta um histórico de evitar os atendimentos psiquiátricos, fato que por vezes pode levar a um déficit de treinamento e capacitação para tais casos, devido à falta de adesão. Em outro estudo, profissionais de saúde envolvidos demonstraram preconceitos e descaso ao atendimento de usuários psiquiátricos, à medida que priorizam questões biológicas e físicas ${ }^{17}$.

Com base nas informações sobre o ocorrido, o médico regulador decidirá sobre o envio de uma Unidade de Suporte Avançado de Vida - responsáveis por urgências traumáticas e clínicas com risco de morte - ou de uma Unidade de Suporte Básico de Vida - que prestam atendimento às situações de menor risco ${ }^{6}$. Portanto, é imprescindível o relato preciso e adequado das informações pelo solicitante ao técnico auxiliar de regulação médica, desprovendo-se de expressões genéricas que dificultem o estabelecimento de uma hipótese diagnóstica, visto que, assim, o tempo decorrido até o envio da ambulância e início das intervenções é reduzido, estando diretamente relacionado a uma maior sobrevida da vítima em situações como a de uma parada cardiorrespiratória ${ }^{20}$. O ideal é que se informe o nome do solicitante, um número de telefone para contato, endereço da ocorrência, estado da vítima, número de vítimas e capacidade de prestar primeiros socorros ${ }^{6}$, sendo que mais da metade dos entrevistados responderam corretamente este quesito. Apesar do fato de que tais informações sejam, necessariamente, questionadas ao solicitante, a ciência de sua importância e seu conhecimento prévio torna a ligação mais objetiva e rápida. No estudo de Fernandes et al 
$(2014)^{6}$ nenhum estudante do curso de medicina entrevistado pelo autor soube responder espontânea e corretamente a todas as informações necessárias a serem passadas.

Vieira e Mussi $(2008)^{21}$ destacaram a relevância de se proporcionar a comunidade educação para que possa reconhecer suas responsabilidades ao se acionar o serviço, além de capacitação para reconhecer rápida e corretamente as circunstâncias que requerem o atendimento do SAMU. Para tanto, propõem programas de treinamento a leigos em instituições públicas como: escolas, empresas, igrejas e centros de saúde, além da distribuição de cartilhas educativas. Outro ponto importante, segundo as autoras, seria a realização de campanhas públicas de conscientização da população sobre o funcionamento e uso adequado do aparelho.

\section{CONCLUSÃO}

Conclui-se que, de forma geral, a população dos três municípios abrangidos apresenta certo grau de conhecimento sobre o SAMU, com relações pouco significantes entre o conhecimento detido e a atuação ou não do serviço em seu município. No entanto, o bom desempenho geral dos entrevistados não deve ser tido como suficiente, uma vez que qualquer erro ou delonga na solicitação do serviço de urgência pode trazer gastos desnecessários e, principalmente, sequelas permanentes ou mesmo a morte. Outro ponto importante é que muitos não detinham dados corretos sobre a presença ou atuação do SAMU em sua cidade, o que poderia levar, por exemplo, ao não acionamento do serviço onde este é atuante e, consequentemente, a não prestação do socorro mais capacitado disponível.

Dessa forma, sugere-se a aplicação de campanhas públicas informativas e de conscientização sobre o uso correto e pertinente do SAMU, utilizando-se de mídias locais e regionais para ampla disseminação de conhecimentos tão fundamentais para momentos em que a vulnerabilidade pode atrapalhar a tomada de decisões, que necessita de rapidez e precisão. Tal ação deve ser proposta mesmo em cidades sem cobertura do serviço, como Pirajuba, uma vez que em casos de viagens ou mudança, todo indivíduo estará capaz de utilizar o aparelho para o salvamento de vidas.

\section{REFERÊNCIAS}

1. Scarpelini S. A organização do atendimento às urgências e trauma. Medicina (Ribeirão Preto) 2007; 40(3):315-20.

2. Martins PPS, Prado ML. Enfermagem e Serviço de Atendimento Pré-Hospitalar: descaminhos e perspectivas. Rev Bras Enferm, Brasília (DF) 2003; 56(1):71-5.

3. Almeida PMV, Dell'Acqua MCQ, Cyrino CMS, Juliani CMCM, PalharES VC, Pavelqueires S. Análise dos atendimentos do SAMU 192: Componente móvel da rede de atenção às urgências e emergências. Esc Anna Nery. 2016; 20(2):289-95.

4. BRASIL. Ministério da Saúde. Política Nacional de Atenção às Urgências. $3^{\mathrm{a}}$ ed. Brasília (DF), 2006. Disponível em: <http://bvsms.saude.gov.br/bvs/ publicaçoes/politica_nacional_atencao_urgencias_3ed.p df>. Acesso em: 22 nov. 2015.

5. Machado CV, Salvador FGF, O’DwyerI G. Serviço de Atendimento Móvel de Urgência: análise da política brasileira. Rev Saúde Pública 2011; 45(3):519-28.
6. Fernandes CR, Cavalcante SB, Pinheiro JA, Costa JV, Costa PL R, Filho AAM. Conhecimento de Estudantes de Medicina sobre o Funcionamento do Serviço de Atendimento Móvel de Urgência (Samu). Rev bras educ med. 2014; 38(2):253-60.

7. SAGE: Sala de Apoio à Gestão Estratégica. Ministério da Saúde (BR). Disponível em: <http://sage.saude. gov.br/>. Acesso em: 1 nov. 2015.

8. Razzak JA, Kellermann AL. Emergency medical care in developing countries: is it worthwhile? Bull World Health Organ. 2002; 80(11):900-5.

9. Abreu KP. Situações de urgência: visão dos usuários do Serviço de Atendimento Móvel de Urgência de Porto Alegre [trabalho de conclusão do curso]. Universidade Federal do Rio Grande do Sul; 2009.

10. BRASIL. Ministério da Saúde. Informações de Saúde. Datasus. Disponível em: <http://datasus.saude.gov.br/ projetos/52-samu>. Acesso em: 1 nov. 2015.

11. Veronese AM, Oliveira DLLC, Nast K. Risco de vida e natureza do SAMU: demanda não pertinente e implicações para a enfermagem. Rev Gaúcha Enferm. 2012; 33(4):142-8.

12. Berg RA, Hemphill R, Abella BS, Aufderheide TP, Cave DM, Hazinski MF, et al. Part 5: adult basic life support 2010 American Heart Association Guidelines for Cardiopulmonary Resuscitation and Emergency Cardiovascular Care. Circulation. 2010; 122(18 Suppl3):685-705.

13. Horta BL, Pereira GAR, Silva AB, Resende BHA, Rosenthal, RM, Comim C, et al. Conhecimento do estudante de Medicina sobre o Serviço de atendimento móvel de Urgência. Rev AMRIGS. 2011; 55(1):20-4.

14. Pontes-Neto OM, Silva GS, Feitosa MR, de Figueiredo NL, Fiorot JA Jr, Rocha TN, et al. StrokeAwareness in Brazil: Alarming Results in a Community-Based Study. Stroke. 2008; 39(2):292-6.

15. BRASIL. SÃO PAULO. Lei complementar $n^{\circ} 1.257$, de 06 de janeiro de 2015. Institui o Código estadual de proteção contra Incêndios e Emergências e dá providências correlatas. Disponível em: <http://www.al. sp.gov.br/repositorio/legislacao/lei.complementar/2015/1 ei.complementar-1257-06.01.2015.html>. Acesso em: 13 dez. 2016.

16. Marques GQ. Acesso e utilização do serviço de atendimento móvel de urgência de Porto Alegre por usuários com demandas clínicas [tese]. Porto Alegre: Escola de Enfermagem, Universidade Federal do Rio Grande do Sul; 2010.

17. Giglio-Jacquemot A. Urgências e emergências em saúde: perspectivas de profissionais e usuários. Rio de Janeiro: Fiocruz, 2005.139 p.

18. Cochlar CA. Investigação do grau de conhecimento sobre a atuação do serviço de atendimento móvel de urgência (SAMU) entre os usuários da triagem do hospital de pronto socorro de Canoas [dissertação]. Canoas: Universidade Luterana do Brasil; 2007.

19. Jardim KFSB. O Serviço de Ambulatorial Móvel de Urgência (SAMU) no contexto da reforma psiquiátrica: em análise a experiência de Aracajú/SE. Natal [dissertação]. Natal: Universidade Federal do Rio Grande do Norte; 2008.

20. Berdowski J, Beekhuis F, Zwinderman AH, Tijssen JGP, Koster RW. Importance of the first link: description and recognition of an out-of-hospital cardiac 


\section{CONFLITO DE INTERESSES}

Os autores declaram não haver conflitos de interesse.

\section{AUTOR PARA CORRESPONDÊNCIA}

Marlene Cabral Coimbra da Cruz

mcoimbracruz@gmail.com

Submetido em 18/03/2017

Aceito em 02/05/2017 